\title{
¿Están preparadas las ciencias veterinarias y zootécnicas para el futuro?: una visión desde Colombia
}

\section{¿Are the colombian zootechnics and veterinary sciences prepare for the future?: A vision from Colombia}

\author{
Fernando Nassar-Montoya, M.Sc. \\ Fundación Universitaria San Martín, Facultad de Medicina Veterinaria y Zootecnia. Bogotá, Colombia.
} Correspondencia: fernando.nassar@sanmartin.edu.co

Recibido: Enero de 2011; Aceptado: Diciembre de 2011.

\begin{abstract}
RESUMEN
Se hace revisión de literatura de los ámbitos de aplicación de la medicina veterinaria y la zootecnia con el objetivo de entender su significado y acción en la sociedad actual y futura, con énfasis en la realidad colombiana. Se muestra cómo estas ciencias tienen competencias y responsabilidades importantes en diversos sectores como el ambiental, social, una salud y no únicamente en el agropecuario. La crisis ambiental y el gran dinamismo que caracteriza el mundo contemporáneo, han creado nuevas necesidades que representan una gran oportunidad para las ciencias veterinarias y zootécnicas, si éstas logran responder a los nuevos retos y aprovechar su rol natural en los sectores que ahora les competen. Su institucionalidad y hegemonía dependerá de su capacidad de adaptación, flexibilidad y liderazgo. Por lo tanto, es pertinente que las ciencias veterinarias y zootécnicas colombianas realicen una prospección colectiva disciplinaria para entender los nuevos retos y les permita prepararse para el futuro.
\end{abstract}

Palabras clave: Ciencias agrícolas, competencia profesional, medicina veterinaria, servicios veterinarios, zootecnia (Fuente:CAB).

\begin{abstract}
A review of the Veterinary Medicine and Zootechnics is done aiming to understand their significance and performance in the current and future society, with main focus in the Colombian reality. The review shows the competences and responsibilities that these sciences have in diverse sectors such as the environmental, social and health, area, and not just in agriculture. The environmental crisis and the dynamics of the modern world have created new needs that represent a great opportunity for the Veterinary sciences and Zootechnics, if they can respond to new challenges and make use of their natural role in the sectors that now are of their competence. Their institutionally and hegemony will depend on their adaptation, flexibility and leadership capacity. Therefore, this is pertinent for the Zootechnics and Veterinary sciences to perform a collective disciplinary prospection to understand new challenges and prepare for the future.
\end{abstract}

Key words: Agricultural sciences, occupational qualifications, veterinary medicine, veterinary services, zootechnics (Source:CAB). 


\section{INTRODUCCIÓN}

La prospección global de las ciencias veterinarias se ha hecho principalmente con base en "para qué y cómo" mediante estudios orientados a entender el mercado de los servicios veterinarios e identificando necesidades técnicas para su aplicación en salud pública, producción de alimentos e investigación biomédica; quizás subestimando el "por qué", a pesar que algunos autores han llamado la atención sobre la necesidad de hacer la resignificación profunda de sus profesiones para responder a las necesidades actuales y futuras de la humanidad $(1,2)$.

En un editorial de la Revista MVZ Córdoba (3), se hacía una pregunta básica para dar inicio a la discusión teórica: "¿Sabemos qué queremos de las ciencias veterinarias y zootécnicas de Colombia y qué necesita el país de éstas?". El propósito era generar un diálogo no solamente a partir del final, es decir, sobre la calidad del producto del servicio veterinario y zootécnico como resultado del cumplimiento de las demandas de la sociedad; sino desde el principio: la identificación propia de lo que es y debería ser la medicina veterinaria y la zootecnia en el contexto local y global. Se visualizaba en el diálogo colectivo nacional una oportunidad para que éstas contribuyeran activamente, como líderes teóricos y prácticos, al destino del país; ya que si bien hay propuestas y acciones individuales que contribuyen a la proyección al nivel nacional como global, parecería que se careciera de una visión integradora multidisciplinaria e intersectorial que habría debilitado su hegemonía e institucionalidad (4).

Lógicamente la construcción colombiana de un significado para las ciencias veterinarias y zootécnicas debe ser coherente con el pensamiento global, pues la ciencia es universal, aunque su aplicación tiene que ser lo suficientemente flexible para adaptarse a la diversidad de las necesidades locales. Es decir, hay que tender por una ciencia robusta conceptualmente pero con alta capacidad para visualizar, proponer y responder a las necesidades de la sociedad y el planeta. De la capacidad que demuestren estas ciencias para responder, dependerá su destino (2).

Con el ánimo de contribuir a la construcción del significado y aplicación de las ciencias veterinarias y zootécnicas con énfasis en la realidad nacional colombiana, en el presente artículo se hace una revisión general de los sectores con los que están íntimamente relacionados en la actualidad, y los cuales deberían contemplarse en el análisis de lo que es y debería ser su papel presente y futuro; no sólo desde el saber y la técnica profesional aplicados, sino como actores analíticos desde el conocimiento científico con compromiso por el bien público y el progreso sostenible ambiental y social.

\section{Contexto de aplicación de las ciencias veterinarias y zootécnicas}

Producción de Alimentos. La seguridad alimentaria mundial está pasando por una serie de retos debidos a los cambios de paradigmas productivos por los nuevos requerimientos sociales, ambientales y de bienestar animal. Algunos de los factores importantes para entender las perspectivas actuales y futuras de las ciencias veterinarias y zootécnicas, son:

\section{Variaciones en las expectativas demográficas mundiales. Las expectativas de las necesidades para la producción de alimentos mundiales para el 2050 han variado, debido al cambio en las tasas de crecimiento demográfico previstas anteriormente, con un efecto heterogéneo entre las regiones (5).}

Impacto ambiental. La producción de alimentos tiene un alto impacto ambiental por deforestación, contaminación de aguas y suelos, degradación del suelo y, la coexistenciacompetencia con la flora y fauna silvestres (6). Por ejemplo, para el caso de Colombia se estima que al año 1998 se había deforestado el 35\% del área total (sin incluir las sabanas naturales) debido a la agricultura (32\%) y pastoreo de ganado (68\%) (7). Por lo tanto, es necesario identificar e implementar nuevas opciones de producción animal más sostenibles y más eficientes (6).

Mejoramiento genético y biotecnologías. El desarrollo de animales más especializados con un objeto productivo, incluye el manejo de tecnologías reproductivas, la manipulación del ácido nucléico y la fusión de células intertaxones. Actualmente, hay preocupación por regular el uso de biotecnologías para garantizar productos seguros (8), lo que se evidencia en el Protocolo de Cartagena (9).

Nuevos paradigmas de bioseguridad. Algunos conceptos han ganado campo dentro de las políticas para la producción y consumo de alimentos como: trazabilidad (rastreabilidad) (10), inocuidad (11) y sostenibilidad (6). Éstos redefinen los procesos de producción, transporte, comercialización y consumo; lo que repercute en la implementación de políticas de calidad. En Colombia, los documentos Conpes 
3376 de 2005, 3458 de 2007 13) y 3468 de 2007 (12-14), enmarcan las políticas sanitarias y de inocuidad para las cadenas de la carne y leche bovina, la cadena porcina y la cadena avícola, respectivamente.

Globalización y nuevos paradigmas comerciales. La intensificación del comercio internacional ha derivado en el lógico aumento en la preocupación sobre la movilización de los productos agropecuarios, lo que ha generado la firma de acuerdos internacionales (15) y de medidas para garantizar la inocuidad de los alimentos, la salud de los animales y la preservación de los vegetales (16). El marco de desarrollo de Colombia para adecuarse a las exigencias sanitarias del comercio internacional se consigna en el documento Conpes 3375 de 2005 (17).

Nuevos paradigmas en el bienestar animal. La calidad de vida de los animales en las granjas y fincas (18) ha empezado a interesar a los consumidores y productores a nivel mundial, aunque parecería haber mayor preocupación en los países desarrollados occidentales; ya que por ejemplo el bienestar animal es un objetivo primordial en la Política Agraria Común europea. Pero más allá, es un momento coyuntural para que la veterinaria y la zootecnia como ciencias, retomen lo que fue su olvido primordial: entender el animal como el pilar fundamental de su conocimiento y no sólo como una máquina productiva, lo que subestima la relación bienestar-salud (4).

Producción de biocombustibles. Ésta puede competir con la producción de alimentos y distorsionar la oferta y el precio. El impacto que tiene la carencia e incremento de los precios de las materias primas de los insumos para animales, repercute en los de los productos de las cadenas de lácteos y cárnicos. El efecto negativo es grande, ya que se estima que en Colombia en las estructuras de costos de las producciones pecuarias, los alimentos representan la mayoría del valor de producción: en la porcicultura aproximadamente el $75 \%$ del total en el ciclo completo, en la avicultura $66 \%$ y en la piscicultura (por ejemplo, cachama y tilapia) $67-80 \%(19-21)$.

Bienes y servicios. En Colombia en la actualidad, se ponderan como sectores socioeconómicos prioritarios del sector agropecuario, la producción de las Cadenas Alimenticias (cárnica y láctea), del Cuero, Calzado y Marroquinería y, Farmacéutica y Medicamentos (22). La veterinaria y la zootecnia han estado tradicionalmente vinculadas a las cadenas cárnica y láctea, pero interesantemente, si se miran los documentos de la Agenda Interna (22), entre las debilidades de la Cadena del Cuero, Calzado y Marroquinera están precisamente elementos relacionados directamente con el ejercicio de estas profesiones "Considerar la piel como un subproducto de la carne" que lógicamente se relaciona con el "Mal manejo del ganado en los hatos, restándole calidad a las pieles".

El papel de las ciencias veterinarias y zootécnicas en la industria farmacéutica debe ser de alta importancia, si se considera que además de su contribución al crecimiento económico del sector, tiene responsabilidad directa en la salud animal y pública; hecho que se enfatiza por el papel que juega el cambio y adaptación de patógenos, incluyendo la aparición de microbios resistentes a los tratamientos, en la emersión de enfermedades infecciosas (23). También, un efecto muy importante y que ha sido poco estudiado en el país, es la bioacumulación de sustancias en las cadenas alimenticias que pueden generar alteraciones morfológicas, fisiológicas y disrupción endocrina en algunas especies, incluyendo al ser humano (24).

Adicionalmente, la veterinaria y la zootecnia están íntimamente relacionadas con el desarrollo de otros sectores de relevancia socioeconómica potencial y que no han sido suficientemente definidos dentro de las cuentas económicas colombianas actuales (25), como lo son las cadenas de animales de trabajo, recreación y compañía. Éstos incluyen los zoológicos, acuarios y zoocriaderos.

Bienestar y progreso social. El veterinario y el zootecnista se constituyen en moderadores naturales del vínculo de la sociedad con los animales. Según Wilson (26), el ser humano tiene una tendencia natural a interesarse en otros seres vivos y el grado de comprensión que éste tenga de otros organismos, contribuirá al valor que les dé a éstos y a sí mismo. Si esto es cierto, dentro del Modelo Dirigido por el Conocimiento de la categorización para definir los propósitos de uso de las ciencias (27), el papel del veterinario y zootecnista tiene que trascender de la pura atención del animal, que de por si es parte fundamental de la profesión, para asumir la responsabilidad humanística de liderar las bases culturales con las que la sociedad se relaciona con los animales y el medio ambiente.

La función social de las ciencias veterinarias debe examinarse también dentro de sus aplicaciones en la salud pública. Adicionalmente 
a sus amplias y reconocidas funciones en la seguridad alimentaria y la prevención y control de zoonosis, en la actualidad se ha visto la importancia de que se consideren los beneficios que conlleva el contacto con animales: -"Nadie que mire la evidencia puede dudar que los animales mejoran la calidad de vida del ser humano moderno" (28). Las oportunidades de contribución serían muy altas, si se considera que de acuerdo con De Zubiría (29), la soledad es uno de los más grandes problemas sociales contemporáneos de importancia en salud pública.

Salud. A nivel global se plantea una evolución conceptual en la salud que involucra a muchas disciplinas tradicionalmente ajenas al sector. Es así como otras áreas del conocimiento han, inclusive, ido más allá y empezado a apropiarse del concepto medicina, como lo son medicina ecológica y medicina geológica $(30,31)$. Por lo tanto, más que justificable es perentorio que los nuevos marcos conceptuales rompan la separación tradicional de la salud, para lo cual la práctica e investigación de la veterinaria tiene que contribuir al entendimiento de una medicina única que integre la salud animal, de los ecosistemas y la humana (32).

La ampliación conceptual y aplicada de salud es una respuesta a las nuevas problemáticas modernas que han derivado en la explosión de enfermedades en la fauna silvestre, los animales domésticos y el ser humano; por los cambios ecológicos, el comportamiento de la población humana, el comercio y movilización de personas internacionalmente, la globalización y los cambios en la tecnología e industria, el cambio y adaptación parasitarios, y las fallas en los programas de salud pública (33). Es así como la emersión de las enfermedades zoonóticas es una prioridad compartida de organismos como por ejemplo, la Organización Mundial de la Salud, la Organización Mundial de Sanidad Animal, la Organización de las Naciones Unidas para la Alimentación y la Agricultura, y la Unión Internacional para la Conservación de la Naturaleza (34).

El reto actual sobre la salud para las ciencias veterinarias es inmenso, quizás ahora más grande que nunca si se considera que es un área de conocimiento clave para el entendimiento de la nueva dinámica de los patógenos que involucra fauna silvestre-animal domésticoser humano. De acuerdo a Jones et al (35) el $60.3 \%$ de 355 eventos estudiados de emersión de enfermedades infecciosas en seres humanos correspondieron a zoonosis, de los cuales $71.8 \%$ se originó en la vida silvestre. Estos autores concluyen que la mayor probabilidad de emersión de enfermedades provenientes de la fauna se localiza en los países en desarrollo de las zonas tropicales de Asia, África y Latinoamérica debido a factores socioeconómicos, ambientales y ecológicos. Colombia es uno de los países que estaría bajo mayor riesgo por patógenos de origen en la vida silvestre y por los transmitidos por vectores; pero a excepción de uno pocos casos y de algunas propuestas de algunos grupos de investigación; el país no está preparado para prevenir o $\mathrm{y} / \mathrm{o}$ responder enfermedades emergentes de vida silvestre; y menos a predecir la aparición de nuevos patógenos. Lo más grave, es que la aplicación de las políticas de investigación y acceso a la biodiversidad están entorpeciendo las iniciativas del sector público y privado para generar conocimiento y entender la situación del país.

Biodiversidad y servicios ambientales. Actualmente es muy utilizado el término cambio global, que se refiere a los cambios de la superficie de la tierra y su uso, la declinación global de la biodiversidad, los cambios en la composición de la atmósfera y el cambio climático. Según Walter y Steffen (36) el más importante para los ecosistemas terrestres es el primero debido al incremento de las necesidades de alimento, situación que se agrava por factores como migración, pobreza e inequidad socioeconómica, y debilidad política. Entonces, indudablemente las actividades antropogénicas representan la mayor amenaza para la biodiversidad del planeta y a su vez el deterioro ambiental se presenta como un limitante para el progreso del ser humano (37). Es decir, la conservación es un asunto socioeconómico además de ambiental, por lo cual es prioritario que las ciencias veterinarias y zootécnicas colombianas definan la posición sobre su responsabilidad. En este sentido, es necesario identificar como éstas abordarán factores causantes de la extinción como, pérdida de hábitats terrestresyacuáticos, contaminación, introducción de especies, uso no sostenible de los recursos y cambios climáticos globales (38); sobre todo cuando Colombia se encuentra en el rango de dos hotspots de biodiversidad: Andes Tropicales y Tumbes- Chocó- Magdalena (39). También, tienen que clarificar sus roles en la emersión de enfermedades infecciosas con alto impacto en las poblaciones naturales (40) y en la problemática que representa la amenaza de extinción en que se encuentran algunas especies domésticas (41).

Innovación y Tecnología. En algunos países, como Estados Unidos, hay lineamientos específicos para la investigación en ciencias 
veterinarias que responden a su amplio contexto de aplicación (32). Sin embargo, en Colombia no se ha abordado la temática desde lo disciplinario; sino sectorial, por lo que se han categorizado como ciencias agrarias. El sistema de gestión se organiza por cadenas productivas; considerándose como prioridades de investigación e innovación pecuaria: inocuidad alimentaria; conservación, caracterización y utilización de recursos zoogenéticos para la alimentación; aplicación de biotecnologías en mejoramiento y valor agregado de los sistemas pecuarios e, innovaciones que soporten la modernización de las distintas cadenas $(22,42)$.

Sin embargo, esto posiblemente subestima el potencial investigativo de la veterinaria y la zootecnia en los ámbitos que no están relacionados con los sistemas de producción pecuaria; lo que puede evidenciarse al revisar la Plataforma Scienti de Colciencias (43), en la que se encuentran registrados solamente 78 grupos de estas profesiones, lo que representa $30.1 \%$ de los grupos de ciencias agrarias y $1.8 \%$ del total de los grupos inscritos en Colombia.

En el Sistema Nacional de Indexación y Homologación de Revistas Especializadas de Colombia están registradas 23 revistas en la Categoría A1, de las cuales dos están destinadas a la divulgación de la investigación en la medicina veterinaria y la zootecnia (44). Ambas están indexadas en Thompson Reuters Science Citation Index Expanded: la Revista de MVZ Córdoba, editada por la Universidad de Córdoba, y la Revista Colombiana de Ciencias Pecuarias, editada por la Universidad de Antioquia. Éstas indudablemente son una fuente importante para entender el estado de la investigación de las ciencias veterinarias y zootécnicas colombianas e identificar necesidades hacía el futuro.

\section{Institucionalidad de las ciencias veterinarias y zootécnicas en Colombia}

La práctica de la medicina veterinaria y la zootecnia en Colombia. Ha sido descrita por García Alzate y Parra López (45). Hay un marco histórico real que le confiere institucionalidad, más allá de la normatividad que ampara y define su ejercicio (46-48).

Sin embargo, las ciencias veterinarias $y$ zootécnicas colombianas se encuentran en un momento decisivo en su historia debido a que parecería, por lo menos en algunos campos, se ha alejado de la situación socioeconómica y ambiental actual, y de las acciones de progreso (3). Ésta problemática no sería exclusivamente nacional de acuerdo al siguiente texto de Nielsen
(2) para la veterinaria: "La profesión tiene que aceptar que su cultura actual modelada solamente en la medicina humana, ha dejado de ser funcional.". De acuerdo a Willis et al (49), la veterinaria pasa por un momento de transición debido a los cambios que están sucediendo en la sociedad y el planeta.

Actualmente hay un replanteamiento permanente de los campos del conocimiento que hace dudar de la hegemonía tradicional que pudiera tener cualquier ciencia o disciplina. De esta manera, el médico veterinario y el zootecnista se ven abocados a competir con otras profesiones que los podrán reemplazar en aquellos ámbitos en los cuales no muestren la adaptación, flexibilidad, desarrollo y liderazgo suficientes para responder a las necesidades de la sociedad en los diferentes sectores que les competen $(2,4)$.

Perspectivas al futuro. La revisión realizada muestra que la medicina veterinaria y la zootecnia son sensibles a los cambios socioeconómicos y ambientales que ocurren al nivel nacional y global. Esto representa una amenaza para su institucionalidad y hegemonía, pero también una gran oportunidad de desarrollo y de extensión en sectores no tradicionales para estas ciencias. Su éxito dependerá de su capacidad de adaptación y flexibilidad para entender los nuevos retos y responder a los paradigmas modernos de calidad, equidad y sostenibilidad; la capacidad de articulación con otras profesiones y saberes $y$, de la conceptualización de la ciencia, la investigación y su aplicación en Una salud (comprendida como la integración de la salud animal, humana y de los ecosistemas), bioseguridad, seguridad alimentaria, salud-bienestar-producción animal, animal-bienestar humano, biodiversidad-usoconservación, producción-sostenibilidad y sistema de producción animal.

Por lo tanto, cuando se observa el potencial de aplicación de las ciencias veterinarias y zootécnicas que trasciende del sector agropecuario; se puede decir que es necesario pensar en un plan de desarrollo disciplinar nacional; pues el sectorial no tendrá la sensibilidad suficiente para comprender su complejidad. Desde este punto de vista, un plan disciplinar tendría que afirmar ante el país una unidad e identidad, respetando la diversidad de pensamiento, disciplina y cultura; caracterizar y profundizar el significado del humanismo para las ciencias veterinarias y zootécnicas e impulsarlo para estimular el ejercicio profesional ético y con calidad y, el reconocimiento científico, social, económico y político de las profesiones. Además, éste será pertinente para el país, si 
logra ser indicador para el progreso dentro del contexto nacional mediante la identificación de prioridades de investigación en las ciencias veterinarias y zootécnicas para garantizar que el conocimiento científico y tecnológico contribuya a responder ante las necesidades que presentan los diversos sectores de influencia.

Pero sobre todo, se requiere de líderes teóricos, por lo que es una excelente noticia la creación de la Academia Colombiana de Ciencias Veterinarias en el año 2005, para la reflexión sobre los fundamentos y paradigmas de su ciencia, principalmente en lo concerniente a las relaciones ser humano-animal-ecosistema $y$, así promover actitudes activas para que la veterinaria y la zootecnia sean profesiones líderes conceptuales y aplicados de la sociedad.
En 2011 se presenta una buena oportunidad para promover la proyección disciplinaria en el país y otras partes del mundo, debido a que se está celebrando globalmente los 250 años de la medicina veterinaria. Es decir, aprovechar una tradición teórica y práctica que pesa para mirar hacia el futuro, en momento propicio para reinventar la ciencia, desde su significado de lo que es y lo que debería ser, más allá de aspectos puramente técnicos. Porque hay que confesar que éstos últimos son los que menos deben importar, ya que tanto la veterinaria y la zootecnia desde este punto de vista, han avanzado a la par de las innovaciones tecnológicas junto con otras ciencias.

\section{Agradecimientos}

Victoria Pereira-Bengoa por la revisión y comentarios al manuscrito.

\section{REFERENCIAS}

1. Nomisma. VET 2020 Development of European Educational Strategies: Design of Veterinarian Profiles Identified by Market Needs for the Year 2020. Bologna: Nomisma; 2002.

2. Nielsen OT. Is the veterinary profession losing its way?. Can Vet J 2001; 41:439-445.

3. Nassar-Montoya F. Un camino para las ciencias veterinarias y zootécnicas colombianas. Editorial. Rev MVZ Córdoba 2008; 13(1):1107-1109.

4. Nassar-Montoya F. Hacia el entendimiento del significado de la veterinaria para Colombia. Revista Academia Colombiana de Ciencias Veterinarias 2010; 1(3):49-60.

5. FAO. World agriculture towards 2030/2050: Prospects for food, nutrition, agriculture and major commodity groups; Interim report. Rome: FAO; 2006.

6. Steinfeld $H$, Gerber $P$, Wassenaar $T$, Castel V, Rosales M, de Haan C. Livestock's Long Shadow; environmental issues and options. Rome: LEAD/FAO; 2006.

7. Etter A, McAlpine C, Wilson K, Phinn $\mathrm{S}$, Possingham $\mathrm{H}$. Regional patterns of agricultural land use and deforestation in Colombia. Agr Ecosyst Environ 2006; 114:369-386.
8. CAC/GL 44-2003, Enmienda 1 - 2008. [Fecha de acceso 28 de febrero de 2011]; URL disponible en: http://www. codexalimentarius.net/web/more_info. jsp?id_sta $=10007$

9. Secretaría del Convenio sobre la Diversidad Biológica. Protocolo de Cartagena sobre Seguridad de la Biotecnología del Convenio sobre la Diversidad Biológica. Montreal: Secretaría del Convenio sobre la Diversidad Biológica; 2000.

10. CAC/GL 60-2006. [Fecha de acceso 28 de febrero de 2011]; URL disponible en: http://www.codexalimentarius.net/web/ more_info.jsp?id_sta $=10603$

11. CAC/GL 62-2007. [Fecha de acceso 28 de febrero de 2011]; URL disponible en: http://www.codexalimentarius.net/web/ more_info.jsp?id_sta $=10751$

12. Consejo Nacional de Política Económica y Social. Documento Conpes 3376: Política sanitaria y de inocuidad para las cadenas de la carne bovina y de la leche. Bogotá: Departamento Nacional de Planeación; 2005.

13. Consejo Nacional de Política Económica y Social. 2007a. Documento Conpes 3458: Política nacional de sanidad e inocuidad para la cadena porcícola. Bogotá: Departamento Nacional de Planeación; 2007. 
14. Consejo Nacional de Política Económica y Social. Documento Conpes 3468: Política nacional de sanidad e inocuidad para la cadena avícola. Bogotá: Departamento Nacional de Planeación; 2007.

15. Organización Mundial del Comercio. Agricultura. Ginebra: OMC; 2003. Serie de los Acuerdos de la OMC 3.

16. Organización Mundial del Comercio. Medidas sanitarias y fitosanitarias. Ginebra: OMC; 2000. Serie de los Acuerdos de la OMC 4.

17. Consejo Nacional de Política Económica y Social. Documento Conpes 3375: Política nacional de sanidad agropecuaria e inocuidad de alimentos para el sistema de medidas sanitarias y fitosanitarias. Bogotá: Departamento Nacional de Planeación; 2005.

18. Five Freedoms. [en línea] 2009 April 19 [fecha de acceso 1 de mayo de 2009]. URL disponible en: http://www.fawc.org.uk/ freedoms.htm.

19. Informe de los proyectos de inversión desarrollados durante el primer semestre de 2010. [en línea] 2010 [fecha de acceso 19 de enero de 2011]. URL disponible en: http://www.porcicol.org.co/ dataFiles/costos/2010/informes/primer_ semestre_2010.pdf.

20. Ministerio de Agricultura y Desarrollo Rural. La cadena de cereales, alimentos balanceados para animales, avicultura y porcicultura en Colombia una mirada global de su estructura y dinámica 19912005. Bogotá: Ministerio de Agricultura y Desarrollo Rural; 2005.

21. Ministerio de Agricultura y Desarrollo Rural. La cadena de la piscicultura en Colombia: una mirada global de su estructura y dinámica 1991-2005. Bogotá: Ministerio de Agricultura y Desarrollo Rural; 2005.

22. Departamento Nacional de Planeación. Agenda Interna para la Productividad y la Competitividad, Bogotá: Departamento Nacional de Planeación; 2008.

23. Morens DM, Folkers GK, Fauci AS. The challenge of emerging and re-emerging infectious diseases. Nature 2004; 430: 242-249.
24. Comprendo Project. [en línea] 2006 november 24 [fecha de acceso 10 de enero de 2011]. URL disponible en: http://www. comprendo-project.org/main1024.html

25. Clasificación central de productos agropecuaria, silvícola y pesca. [en línea] 2008 [fecha de acceso 1 de enero de 2009]. URL disponible en: http://www.dane.gov. co/files/nomenclaturas/ces/CPC1_1.pdf

26. Wilson EO. Biophilia. Cambridge: Harvard University Press; 1984.\}

27. Weiss $\mathrm{CH}$.. The many meanings of research utilization. Public Admin Rev 1979; 39: 426-431.

28. Kellert SR, Wilson EO. Biophilia hypothesis. Washington: Shearwater Books; 1993.

29. De Zubiría M. Cómo prevenir la soledad, la depresión y el suicidio en los niños y jóvenes. Bogotá: Editorial Santillana; 2007.

30. Weinhold B. Conservation medicine: Combining the best of all worlds. EHP 2003; 111(10):525-529.

31. Skinner HCW, Berger AR. Editors. Geology and Health: Closing the Gap. New York: Oxford University Press; 2003.

32. Committee on the National Needs for Research in Veterinary Science. Critical needs for research in veterinary science. Washington: The National Academies Press; 2005.

33. Morse SS. Factors in the Emergence of Infectious Diseases. Emerg infect dis 1995; 1 (1):7-15.

34. Empres: Emergency prevention system. [en línea] 2011 February 23 [fecha de acceso 2 de marzo de 2011]. URL disponible en: http:// www.fao.org/ag/againfo/programmes/en/ empres/about.html.

35. Jones KE, Patel NG, Levy MA, Storeygard A, Balk D, Gittleman JL et al. Global trends in emerging infectious disease. Nature 2008; 451 (21): 990-993.

36. Walker B, Steffen W. Editors. The Terrestrial Biosphere and Global Change: Implications for Natural and Managed Ecosystems. A Synthesis of GCTE and Related Research. Stockholm: The International Geosphere Biosphere Program; 1997. 
37. PNUMA. Perspectivas del Medio Ambiente Mundial GEO4. Dinamarca: Programa de las Naciones Unidas para el Medio Ambiente; 2007.

38. Chivian E. Editor. Biodiversity: Its importance to Human Health. Cambridge: Center for Health and Global Environment, Harvard Medical School; 2003.

39. Myers $\mathrm{N}$, Mittermeier RA, Mittermeier CG, de Fonseca GA, Kent J. Biodiversity hotspots for conservation priorities. Nature 2000; 403: 853-858.

40. Daszak $P$, Cunningham $A A$, Hyatt $A D$. Emerging infectious diseases of wildlifethreats to biodiversity and human health. Science 2000; 287: 443-449.

41. Pilling D, Rischkowsky B. Editores. La Situación de los Recursos Zoogenéticos Mundiales para la Alimentación y la Agricultura -resumen. Roma: FAO; 2007.

42. Colciencias. Plan estratégico programa nacional de ciencia y tecnologías agropecuarias; bases para una política de promoción de la innovación y el desarrollo tecnológico en Colombia 2005-2015. Bogotá: Colciencias, 2005.

43. Colciencias Scienti Colombia. [en línea] 2011 [fecha de acceso 19 de enero de 2011]. URL disponible en: http://201.234.78.173:8083/ciencia-war/ BusquedaGrupoXArea.do.

44. Colciencias Publindex. [en línea] 2011 [fecha de acceso 19 de enero de 2011]. URL disponible en: http://201.234.78.173:8084/ publindex/EnRevista/detalle.do? tableName $=$ enArticulo.table. allDetalle\& tableAction $=$ reset\&cod_revista_c $=67$
45. García Alzate H, Parra López LG (compiladores redactores). Medicina veterinaria y zootecnia en Colombia: trayectoria durante el siglo XX y perspectivas para el siglo XXI. Bogotá: Fundación Edivez; 2002.

46. Ley $73 / 1985$ de 8 de octubre, del ejercicio de las profesiones de Medicina y Veterinaria, Medicina Veterinaria y Zootecnia y Zootecnia. (Diario Oficial República de Colombia, número 37186, de 11-10-1985).

47. Decreto $1122 / 1988$ de 10 de junio, por el cual se reglamenta la Ley 73 de 1985 sobre el ejercicio de las profesiones de Medicina Veterinaria, Medicina Veterinaria y Zootecnia y Zootecnia. (Diario Oficial República de Colombia, número 38375, de 14-05-1988).

48. Ley $576 / 2000$ de 15 de febrero, por la cual se expide el Código de Ética para el ejercicio profesional de la Medicina Veterinaria, la Medicina Veterinaria y Zootecnia y Zootecnia. (Diario Oficial República de Colombia, número 43897, de 17-2-2000).

49. Willis NG, Monroe FA, Potworowski A, Halbert G, Evans BR et al. Envisioning the Future of Veterinary Medical Education: The Association of American Veterinary Medical Colleges Foresight Project, Final Report. J Vet Med Educ 2007; 34(1):1-41. 